\title{
Spectra of Harmonic Oscillators with GUP and Extra Dimensions
}

\author{
Benrong Mu, Rong Yu, Dongmei Wang* \\ Physics Teaching and Research Section, College of Medical Technology, Chengdu University of Traditional Chinese Medicine, \\ Chengdu, China \\ Email: benrongmu@cdutcm.edu.cn,19852036@cdutcm.edu.cn, *dongmeiwang@cdutcm.edu.cn
}

How to cite this paper: $M u, B . R ., Y u, R$. and Wang, D.M. (2019) Spectra of Harmonic Oscillators with GUP and Extra Dimensions. Journal of High Energy Physics, Gravitation and Cosmology, 5, 279-290. https://doi.org/10.4236/jhepgc.2019.51015

Received: December 12, 2018

Accepted: January 21, 2019

Published: January 24, 2019

Copyright () 2019 by author(s) and Scientific Research Publishing Inc. This work is licensed under the Creative Commons Attribution International License (CC BY 4.0). http://creativecommons.org/licenses/by/4.0/

\begin{abstract}
In this paper, we address the spectra of simple harmonic oscillators based on the generalized uncertainty principle (GUP) with a Kaluza-Klein compactified extra dimension. We show that in this scenario, to make the results compatible with experiments, the minimal length scale equals to the radius of compact extra dimension.
\end{abstract}

\section{Keywords}

Compactified Extra Dimension, GUP, Harmonic Oscillator

\section{Introduction}

All quantum gravity theories, such as string theory and loop gravity theory, predict that there exits a minimal observable length, which proportional to the Planck length $\ell_{p} \sim 10^{-33} \mathrm{~cm}$ [1]-[7]. This length scale can be easily justified by combining black hole physics with quantum uncertainty principle. Quantum mechanics is free of the theory of gravity. However, when the energy scale approaches Planck scale, the effect of quantum gravity cannot be neglected. It means that quantum mechanics should be modified to accommodate the influences from gravity. Or, it implies that we need a gravitational quantum mechanics. The simplest way is to modify the Heisenberg Uncertainty Principle. The uncertainty principle $\Delta x \Delta p \geq 1 / 2$ shows that the large $\Delta p$ could make the position distance $\Delta x$ arbitrarily small. When the gravity is included in the theory, the $\Delta x$ acquires a minimal length, caused by the emerged horizon of mini black hole. To realize this minimal length scale, we introduce a simple model, the so-called the Generalized Uncertainty Principle (GUP). Incorporating GUP into black holes has been discussed in a lot of papers [8] [9] [10] [11] [12]. It is a toy model of quantum gravity theory, which is expected to reveal some features 
of the ultimate theory. Nearly all problems in quantum mechanics could be discussed with this modified commutator to model the system bathed in strong gravitational background. The modified fundamental commutator $(\hbar=c=1)$ is [13]-[25]

$$
[x, p]=\text { if }(p) \text {. }
$$

This new commutator relation includes the different conditions, which could describe the Heisenberg Uncertainty Principle in quantum mechanics by $f(p)=1$ and also includes the nonzero position distance in high energy scale. In our requirement, $f(p)$ is a positive function and which can be expanded in Taylor series by $f(p)=1+\beta p^{2}+\mathcal{O}\left(p^{4}\right)$, and $\beta=\beta_{0} \ell_{p}^{2}=\beta_{0} / M_{p}^{2}$. Our previous work had introduced the running constant $\beta_{0}$ of $f(p)$, which will varies with energy scales and be combined with extra dimensions [26]. It leads us to construct the low energy effective theory which contains some quantum gravity phenomenons in intermediate energy scale. In GUP model, we choose the first two terms such that

$$
[x, p]=i\left(1+\beta p^{2}\right),
$$

Note that this formalism is special case of generalized modification that $[x, p]=i \hbar\left(1+\alpha x^{2}+\beta p^{2}\right)$. Since the momentum has not minimal scale, it is restricted by choosing $\alpha=0$. Therefore, this special expression gives the generalized uncertainty principle (GUP)

$$
\Delta x \Delta p \geq \frac{1}{2}\left[1+\beta(\Delta p)^{2}\right] .
$$

Generalizing the commutator to high dimensional space and expressing it as tensor formalism, one obtains that ${ }^{1}$

$$
\left[x_{i}, p_{j}\right]=i\left(\delta_{i j}+\beta \delta_{i j} p^{2}+2 \beta p_{i} p_{j}\right),
$$

and the correspondent higher dimensional GUP model is

$$
\Delta x_{i} \Delta p_{i} \geq \frac{1}{2}\left[1+\beta\left((\Delta p)^{2}+\langle p\rangle^{2}\right)+2 \beta\left(\Delta p_{i}^{2}+\left\langle p_{i}\right\rangle^{2}\right)\right]
$$

where $p^{2}=\sum p_{i} p_{i}$. This uncertainty principle easily deduces the minimal observable length that $\Delta\left(x_{i}\right)_{\min } \sim \sqrt{\beta_{0}} \ell_{p}$ for every direction.

In [26], the energy levels of a free particle including an extra dimension was discussed. The results showed that the minimum observable length equals to the compactification radius of the extra dimension. However, if the particle experiences an non-vanishing potential, what is the result likely to be? In this paper, we extend our discussion to the harmonic oscillator in the extra dimension and consider the GUP's modified energy levels. The result is agree with previous work. The present works of extra dimensions can be refereed in [27]-[36].

This paper is organized as follows. In Section 2, the perturbation method based on GUP is briefly reviewed. In Section 3, two dimensional harmonic oscillator including an compact extra dimension is discussed, and the energy spec-

${ }^{1}$ The generic expression is $\left[x_{i}, p_{j}\right]=i\left(\delta_{i j}+\beta \delta_{i j} p^{2}+\beta^{\prime} p_{i} p_{j}\right)$. We choose $\beta^{\prime}=2 \beta$. 
trum is obtained for the large and the small extra dimension. In Section 4, based the perturbation method and GUP, the energy spectrum including quantum gravitational effect is obtained. In Section 5, we summarize the conclusion obtained in the Section 4.

\section{The Perturbation Method Based on GUP}

In our scenario, we require the wave function satisfies the periodicity $\psi(x, y)=\psi(x, y+2 \pi \rho)$, where $x$ represents the usual Euclidean space and $y$ represents the compactified space. Obviously, the function can be separated into two parts $\psi(x, \theta)=\phi(x) \Theta(\theta)$, where $\Theta$ represents the wave function in compactified space, and we have $\Theta(\theta)=\Theta(\theta+2 \pi)$. Therefore, the energy spectrum of Euclidean and compactified space can be obtained respectively. After combining these two parts, we can find the total energy of the system. Following this step, we use the minimal length uncertainty relations, GUP specially, to rewrite the wave function. The modified operators are given by

$$
\begin{gathered}
x_{i}=x_{0 i}, \\
p_{i}=p_{0 i}\left(1+\beta p_{0}^{2}\right), \quad p_{0 i}=-i \frac{\mathrm{d}}{\mathrm{d} x_{0 i}},
\end{gathered}
$$

where $p_{0}^{2}=\sum p_{0 i} p_{0 i}$ and $\left[x_{0 i}, p_{0 j}\right]=i \hbar \delta_{i j}$ are usual canonical operators. The unperturbed Schrödinger equation reads

$$
\left[\frac{p^{2}}{2 m}+V(\boldsymbol{x})\right] \psi=E \psi .
$$

Modifying the commutator relation, we can obtain the new Hamiltonian of this quantum system

$$
H=H_{0}+H_{1}=\frac{p_{0}^{2}}{2 m}+V(\boldsymbol{x})+\frac{\beta}{m} p_{0}^{4},
$$

the first-order corrections to the energy eigenvalues are given as

$$
\begin{aligned}
E_{n}^{(1)} & =\frac{\beta}{m}\left\langle\psi\left|p_{0}^{4}\right| \psi\right\rangle=4 m \beta\left\langle\left(E_{n}^{(0)}-V\right)^{2}\right\rangle, \\
& =4 m \beta\left[\left(E_{n}^{(0)}\right)^{2}-2 E_{n}^{(0)}\langle V\rangle+\left\langle V^{2}\right\rangle\right] .
\end{aligned}
$$

\section{Review of Harmonic Oscillator in Compactified Extra Dimension}

Considering the two dimensional harmonic oscillator which includes one of dimensional Euclidean space and another is compactified extra space, we need to solve the Schrödinger equation

$$
\frac{p^{2}}{2 m} \psi+\frac{1}{2} m \omega^{2}\left(x^{2}+y^{2}\right) \psi=E \psi
$$

The Schrödinger equation can be solved by the method of separation of variables, $\psi(x, y)=\phi(x) \Theta(y)$, with $\Theta(y+2 \pi \rho)=\Theta(y)$. In this section, we do 
not discuss the effects of GUP in Equation (10), $p_{i}=-i \frac{\partial}{\partial x_{i}}, \quad p^{2}=\sum p_{j} p_{j}$. Only the harmonic oscillator with periodic condition is reviewed in this section. In the next section, we will discuss the GUP modification of Equation (10), and the perturbation method will be applied since it is convenient enough for seeing some features of quantum gravity. Now, we rewrite Equation (10) as

$$
H_{x}^{(0)} \phi(x)=E_{x}^{(0)} \phi(x), \quad H_{y}^{(0)} \Theta(y)=E_{y}^{(0)} \Theta(y), \quad E^{(0)}=E_{x}^{(0)}+E_{y}^{(0)} .
$$

Since the harmonic oscillator lives in the compactified space, the physical meaning of the equation $H_{y}^{(0)} \Theta(y)=E_{y}^{(0)} \Theta(y)$ will be changed. The harmonic oscillator in Euclidean space $x$ only satisfies the boundary condition by $\phi( \pm \infty)=0$. However, it should satisfy $\Theta(\theta)=\Theta(\theta+2 \pi \rho)$ and add an additional restriction for the potential by $V(y)=V(y+2 \pi \rho)$ in the extra dimension $y$, which identifies any two points that the length differ by $2 \pi R$. However, the potential of simple harmonic oscillator does not satisfy this condition, we need to construct a new potential of harmonic oscillator with periodicity. Bezerra and Rego-Monteiro had discussed the harmonic oscillator on a circle and constructed a new wave function with periodic oscillator potential [37]. The momentum operator $G$ in a compactified space is given by [37] [38]

$$
G \rightarrow \frac{\hbar}{i} \frac{\mathrm{d}}{\mathrm{d} \theta}+\alpha, \quad 0 \leq \alpha<1 .
$$

Therefore, the Schrödinger equation in the extra space is constructed with the unitary operator $W \rightarrow \mathrm{e}^{i \theta}$ as

$$
\frac{1}{2 m \rho^{2}} G^{2} \Theta+m \omega^{2} K\left[W+W^{\dagger}\right] \Theta=\epsilon \Theta,
$$

where $\epsilon$ is the eigenvalue of extra space. In Equation (13), the unitary operator $W$ describes the periodic potential in the extra space, the parameter $K$ is used for reducing the Equation (13) to the simple harmonic oscillator when $\rho \rightarrow \infty$.

In order to determine the value of $K$, we set $\alpha=0$ and simplify Equation (13) as

$$
\frac{\hbar^{2}}{2 m \rho^{2}} \frac{\mathrm{d}^{2} \Theta}{\mathrm{d} \theta^{2}}+\left(\epsilon-2 m \omega^{2} K \cos \theta\right) \Theta=0 .
$$

Let $\eta=\theta / 2$, Equation (14) becomes

$$
\frac{\hbar^{2}}{2 m \rho^{2}} \frac{\mathrm{d}^{2} \Theta}{\mathrm{d} \eta^{2}}+\left(4 \epsilon-8 m \omega^{2} K \cos 2 \eta\right) \Theta=0 .
$$

with $\Theta(\eta) \sim \Theta(\eta+\pi)$. Moreover, we define

$$
\theta=2 \eta=\frac{1}{\rho} y+\pi, \quad 0 \leq y \leq \pi \rho,
$$

and set $E=\epsilon+2 m \omega^{2} K$, Equation (15) can be rewritten as

$$
\frac{\hbar^{2}}{2 m} \frac{\mathrm{d}^{2} \Theta}{\mathrm{d} y^{2}}+\left[E-m \omega^{2} \frac{1}{\rho^{2}} K y^{2}\left(\frac{\sin y / 2 \rho}{y / 2 \rho}\right)^{2}\right] \Theta=0
$$


When $\rho \rightarrow \infty$, Equation (17) must reduce to the simple harmonic oscillator. This means the coefficient of $y^{2}$ equals 1 . Then we have

$$
K=\frac{1}{2} \rho^{2} .
$$

Substituting the value of $K$ back into Equation (15), and set $a=8 m \rho^{2} \epsilon / \hbar^{2}$, $q=4 m^{2} \omega^{2} \rho^{4} / \hbar^{2}$, we obtain the Mathieu equation

$$
\frac{\mathrm{d}^{2} \Theta}{\mathrm{d} \eta^{2}}+[a-2 q \cos (2 \eta)] \Theta=0 .
$$

The solutions of Mathieu's equation cannot be expressed in terms of hypergeometric functions. We address two extremal situations in the following discussions.

1) $q \gg 1$ case. This situation corresponds to the large extra dimension. The characteristic values $a_{j}$ of Mathieu function, which represent energy spectrum of harmonic oscillator in the compactified space, are given by [39] [40]

$$
a_{j} \approx-2 q+2 v_{j} \sqrt{q}-\frac{\left(v_{j}^{2}+1\right)}{2^{3}}-\frac{\left(v_{j}^{3}+3 v_{j}\right)}{2^{7} \sqrt{q}}-\frac{\left(5 v_{j}^{4}+34 v_{j}^{2}+9\right)}{2^{12} q}-\cdots
$$

where $v_{j}=2 j+1$ and $q=4 m^{2} \omega^{2} \rho^{4} / \hbar^{2}$. Define [39]

$$
\omega^{\prime}=\frac{2 \hbar}{m \rho^{2}} \sqrt{q},
$$

the spectrum in (15) is given by

$$
4 \epsilon \simeq-\frac{q}{m \rho^{2}}+\left(j+\frac{1}{2}\right) \hbar \omega^{\prime}-\frac{\left(v_{j}^{2}+1\right)}{2^{4} m \rho^{2}}-\frac{\left(v_{j}^{3}+3 v_{j}\right)}{2^{8} m \rho^{2} \sqrt{q}}-\frac{\left(5 v_{j}^{4}+34 v_{j}^{2}+9\right)}{2^{13} m \rho^{2} q}-\cdots
$$

It includes the usual energy spectrum of simple harmonic oscillator and the energy spectrum which comes from the quantization of extra dimension.

2) $q \ll 1$ case. This situation corresponds to the small extra dimension. When $q \ll 1$, the solution to Equation (18) has two branches, one is even, the other is odd [41]. In our model, $\eta$ has the periodicity $\pi$, so we only adopt the even solutions in [41]. We rearrange the notation of the even solutions as follows

$$
\begin{gathered}
\Theta_{l=2 m}(\eta, q) \equiv \sqrt{\frac{2}{\pi}} c e_{2 m}(\eta, q) \equiv \sum_{r=0}^{\infty} A_{2 r}^{(2 m)}(q) \cos [2 r \eta], \\
\Theta_{l=2 m+1}(\eta, q) \equiv \sqrt{\frac{2}{\pi}} S_{2 m+2}(\eta, q) \equiv \sum_{r=0}^{\infty} B_{2 r+2}^{(2 m+2)}(q) \sin [(2 r+2) \eta],
\end{gathered}
$$

where $m=0,1, \cdots$, and $[40]$

$$
\begin{aligned}
& A_{2 m-2 r}^{(2 m)} \simeq B_{2 m-2 r}^{(2 m)} \simeq \frac{(2 m-r-1) !}{r !(2 m-1) !} t^{r}, \quad(r>0,2 m-2 r \geq 0), \\
& A_{2 m+2 r}^{(2 m)} \simeq B_{2 m+2 r}^{(2 m)} \simeq(-1)^{r} \frac{(2 m) !}{r !(r+2 m) !} t^{r}, \quad(r>0,2 m>0),
\end{aligned}
$$

with $t=q / 4$.

The normalization of wave function $\Theta(\eta, q)$ requires the functions $c e_{2 m}$ 
and $s e_{2 m}$ normalized as follows

$$
\int_{0}^{\pi} c e_{2 m}(\eta, q) c e_{2 n}(\eta, q) \mathrm{d} \eta=\int_{0}^{\pi} s e_{2 m}(\eta, q) s e_{2 n}(\eta, q) \mathrm{d} \eta=\frac{\pi}{2} \delta_{m n} .
$$

From Equation (22) and Equation (23), the Mathieu Equation (18) can fall into into two categories

$$
\begin{gathered}
\frac{\mathrm{d}^{2} c e_{2 m}}{\mathrm{~d} \eta^{2}}+\left[a_{2 m}-2 q \cos (2 \eta)\right] c e_{2 m}=0, \\
\frac{\mathrm{d}^{2} s e_{2 m+2}}{\mathrm{~d} \eta^{2}}+\left[b_{2 m+2}-2 q \cos (2 \eta)\right] s e_{2 m+2}=0 .
\end{gathered}
$$

Corresponding to the wave function (22) and (23), we write the energy spectrum as

$$
a_{l=2 m} \equiv a_{2 m}, \quad a_{l=2 m+1} \equiv b_{2 m+2}, \quad m=0,1, \cdots
$$

More specifically, for $m \leq 3$, we have the energy spectrum that [42]

$$
\begin{gathered}
a_{0}=-\frac{1}{2} q^{2}+\frac{7}{128} q^{4}-\frac{29}{2304} q^{6}+\frac{68687}{18874368} q^{8}+\mathcal{O}\left(q^{10}\right) \\
a_{1}=4-\frac{1}{12} q^{2}+\frac{5}{13824} q^{4}-\frac{289}{79626240} q^{6}+\frac{21391}{458647142400} q^{8}+\mathcal{O}\left(q^{10}\right) \\
a_{2}=4+\frac{5}{12} q^{2}-\frac{763}{13824} q^{4}+\frac{1002401}{79626240} q^{6}-\frac{1669068401}{458647142400} q^{8}+\mathcal{O}\left(q^{10}\right), \\
a_{3}=16+\frac{1}{30} q^{2}-\frac{317}{864000} q^{4}-\frac{10049}{2721600000} q^{6}+\mathcal{O}\left(q^{8}\right) \\
a_{4}=16+\frac{1}{30} q^{2}+\frac{433}{864000} q^{4}-\frac{5701}{2721600000} q^{6}+\mathcal{O}\left(q^{8}\right) \\
a_{5}=36+\frac{1}{70} q^{2}+\frac{187}{43904000} q^{4}-\frac{5861633}{92935987200000} q^{6}+\mathcal{O}\left(q^{8}\right) \\
a_{6}=36+\frac{1}{70} q^{2}+\frac{187}{43904000} q^{4}+\frac{6743617}{92935987200000} q^{6}+\mathcal{O}\left(q^{8}\right)
\end{gathered}
$$

Notice that when $l \rightarrow \infty, a_{l}-b_{l}=\mathcal{O}\left(q^{l} / l^{l-1}\right)$. In fact, for $m>3$ and $l=2 m$, we have

$$
a_{l} \approx b_{l} \approx l^{2}+\frac{q^{2}}{2\left(l^{2}-1\right)}+\frac{\left(5 l^{2}+7\right) q^{4}}{32\left(l^{2}-1\right)^{3}\left(l^{2}-4\right)}+\frac{\left(9 l^{4}+58 l^{2}+29\right) q^{6}}{64\left(l^{2}-1\right)^{5}\left(l^{2}-4\right)\left(l^{2}-9\right)}+\cdots
$$

Combining the spectrums in the part of extra dimension and the part of Euclidean space, one can easily find the total energy spectrum of simple harmonic oscillator:

$$
E_{s(j \text { or } l)}^{(0)}=E_{x}^{(0)}+E_{y}^{(0)}=\hbar \omega\left(s+\frac{1}{2}\right)+\frac{\hbar^{2}}{2 m \rho^{2}} \begin{cases}a_{j}, & q \gg 1, \\ a_{l}, & q \ll 1 .\end{cases}
$$

\section{The Energy Spectrum with GUP}

Now, we begin to modify the commutator relations. The Schrödinger equation is 
given by Equation (7), and $p$ is determined by Equation (6). The first-order corrections of the energy eigenvalues are given by Equation (9). We only consider the small extra dimension, that is, $q \ll 1$. Then we rewrite Equation (9) as

$$
E^{(1)}=4 m \beta\left\langle\left(E_{n l}^{(0)}-V\right)^{2}\right\rangle=4 m \beta\left[\left(E_{n l}^{(0)}\right)^{2}-2 E_{n l}^{(0)}\langle V\rangle+\left\langle V^{2}\right\rangle\right] .
$$

From Equation (10) and Equation (18), the potential is

$$
V=\frac{1}{2} m \omega^{2} x^{2}+4 m \omega^{2} \rho^{2} \cos 2 \eta .
$$

Then using Equations (22) (25), we have

$$
\begin{gathered}
\langle V\rangle=\left\langle\frac{1}{2} m \omega^{2} x^{2}\right\rangle+\left\langle 4 m \omega^{2} \rho^{2} \cos 2 \eta\right\rangle \\
=\frac{1}{2} \hbar \omega\left(n+\frac{1}{2}\right)+4 m \omega^{2} \rho^{2} \Lambda_{l}, \\
\left\langle V^{2}\right\rangle=\left\langle\frac{1}{4} m^{2} \omega^{4} x^{4}\right\rangle+\left\langle 4 m^{2} \omega^{4} \rho^{2} x^{2} \cos 2 \eta\right\rangle+\left\langle 16 m^{2} \omega^{4} \rho^{4} \cos ^{2} 2 \eta\right\rangle \\
=\frac{\hbar^{2} \omega^{2}}{16}\left(6 n^{2}+6 n+3\right)+4 m \hbar \omega^{3} \rho^{2} \Lambda_{l}\left(n+\frac{1}{2}\right)+16 m^{2} \omega^{4} \rho^{4} \Xi_{l},
\end{gathered}
$$

where

$$
\begin{gathered}
\Lambda_{l=2 m}=A_{0}^{(2 m)} A_{2}^{(2 m)}+\sum_{r=0}^{\infty} A_{2 r}^{(2 m)} A_{2 r+2}^{(2 m)}, \\
\Lambda_{l=2 m+1}=\sum_{r=0}^{\infty} B_{2 r+2}^{(2 m+2)} B_{2 r+4}^{(2 m+2)}, \\
\Xi_{l=2 m}=\frac{1}{2} A_{0}^{(2 m)} A_{4}^{(2 m)}+\frac{1}{2} \sum_{r=0}^{\infty} A_{2 r}^{(2 m)} A_{2 r+4}^{(2 m)}+\frac{1}{2}, \\
\Xi_{l=2 m+1}=\frac{1}{2} \sum_{r=0}^{\infty} B_{2 r+2}^{(2 m+2)} B_{2 r+6}^{(2 m+2)}+\frac{1}{2},
\end{gathered}
$$

Therefore, we obtain the total energy spectrum

$$
\begin{aligned}
E_{n l}= & E_{n l}^{(0)}+4 m \beta\left\{\left(E_{n l}^{(0)}\right)^{2}-E_{n l}^{(0)}\left[\hbar \omega\left(n+\frac{1}{2}\right)+8 m \omega^{2} \rho^{2} \Lambda_{l}\right]\right. \\
& \left.+\frac{\hbar^{2} \omega^{2}}{16}\left(6 n^{2}+6 n+3\right)+4 m \hbar \omega^{3} \rho^{2} \Lambda_{l}\left(n+\frac{1}{2}\right)+16 m^{2} \omega^{4} \rho^{4} \Xi_{l}\right\},
\end{aligned}
$$

with

$$
E_{n l}^{(0)}=\hbar \omega\left(n+\frac{1}{2}\right)+\frac{\hbar^{2} a_{l}}{2 m \rho^{2}}
$$

Now, for analyzing the result, we should calculate the value of $\Lambda_{l}$ and $\Xi_{l}$. We know that when $q \rightarrow 0, A_{m}^{(m)}=B_{m}^{(m)}=1$. From Equation (24) and Equation (25), we calculate four states' values of $\Lambda_{l}$ and $\Xi_{l}$

$$
\Lambda_{l=0}=-t+\sum_{r=0}^{\infty} \frac{1}{[r !(r+1) !]^{2}}(-t)^{2 r+1},
$$




$$
\begin{aligned}
& \Lambda_{l=1}=\sum_{r=0}^{\infty} \frac{4}{r !(r+1) !(r+2) !(r+3) !}(-t)^{2 r+1}, \\
& \Lambda_{l=2}=2 t+\sum_{r=0}^{\infty} \frac{4}{r !(r+1) ![(r+2) !]^{2}}(-t)^{2 r+1}, \\
& \Lambda_{l=3}=\frac{1}{3} t+\sum_{r=0}^{\infty} \frac{576}{r !(r+1) ![(r+4) !]^{2}}(-t)^{2 r+1},
\end{aligned}
$$

and

$$
\begin{gathered}
\Xi_{l=0}=\frac{1}{8} t^{2}+\frac{1}{2} \sum_{r=0}^{\infty} \frac{1}{[r !(r+2) !]^{2}} t^{2 r+2}+\frac{1}{2}, \\
\Xi_{l=1}=\frac{1}{2} \sum_{r=0}^{\infty} \frac{4}{r ![(r+2) !]^{2}(r+4) !} t^{2 r+2}+\frac{1}{2}, \\
\Xi_{l=2}=-\frac{1}{3} t^{2}+\frac{1}{2} \sum_{r=0}^{\infty} \frac{4}{r ![(r+2) !]^{2}(r+4) !} t^{2 r+2}+\frac{1}{2}, \\
\Xi_{l=3}=-\frac{1}{10} t^{2}+\frac{1}{2} \sum_{r=0}^{\infty} \frac{576}{r !(r+2) !(r+4) !(r+6) !} t^{2 r+2}+\frac{1}{2} .
\end{gathered}
$$

Now, considering Equation (47), and using

$$
\frac{3}{8}\left(E_{n}^{(0)}\right)^{2}=\frac{\hbar^{2} \omega^{2}}{16}\left(6 n^{2}+6 n+\frac{3}{2}\right) \sim \frac{\hbar^{2} \omega^{2}}{16}\left(6 n^{2}+6 n+3\right),
$$

we obtain the asymptotic formalism of total energy spectrum

$$
\begin{aligned}
E_{n l}^{(0)} \simeq & E_{n}^{(0)}\left(1+\frac{3}{2} m \beta E_{n}^{(0)}-16 m^{2} \omega^{2} \beta \rho^{2} \Lambda_{l}\right) \\
& +\left(1+4 m \beta E_{n}^{(0)}+2 \beta \frac{\hbar^{2} a_{l}}{\rho^{2}}-32 m^{2} \omega^{2} \beta \rho^{2} \Lambda_{l}\right) \frac{\hbar^{2} a_{l}}{2 m \rho^{2}} \\
& +64 m^{3} \omega^{4} \beta \rho^{4} \Xi_{l} .
\end{aligned}
$$

In Equation (58), the terms $\beta \rho^{2}$ are small, and which can be neglected. Then Equation (58) should be rewritten as

$$
E_{n l} \simeq E_{n}^{(0)}\left(1+\frac{3}{2} m \beta E_{n}^{(0)}\right)+\left(1+4 m \beta E_{n}^{(0)}+2 \beta \frac{\hbar^{2} a_{l}}{\rho^{2}}\right) \frac{\hbar^{2} a_{l}}{2 m \rho^{2}} .
$$

This expression has same expression with our previous work [26]. According to the analysis in [26], for the small extra space, the second term is larger than the first term since it has the term $1 / \rho^{2}$. In the high energy case, the leading order of spectrum, which includes the first excited state of extra dimension effect, can be written as

$$
E_{11} \simeq\left(1+2 \beta \frac{\hbar^{2} a_{1}}{\rho^{2}}\right) \frac{\hbar^{2} a_{1}}{2 m \rho^{2}}
$$

In an intermediate energy scale where the gravity becomes important, we have

$$
M_{I} \sim M_{P} / \sqrt{\beta_{0}} .
$$


It is easy to find

$$
M_{I} \simeq E_{11} \simeq\left(1+2 \frac{\hbar^{2} a_{1}}{M_{I}^{2} \rho^{2}}\right) \frac{\hbar^{2} a_{1}}{2 M_{I} \rho^{2}}
$$

With simple calculation, we obtain

$$
\frac{\hbar^{2} a_{1}}{2 M_{I}^{2} \rho^{2}}+\left(\frac{\hbar^{2} a_{1}}{M_{I}^{2} \rho^{2}}\right)^{2} \sim 1
$$

this gives us the surprise relation

$$
M_{I} \rho \sim 1 \text { and } \Delta x_{\min }=\sqrt{\beta}=\sqrt{\beta_{0}} \ell_{p} \sim \rho .
$$

\section{Conclusion}

In summary, we have discussed the GUP effect of harmonic oscillator in the compactified extra dimension. We use the modified momentum operator and modified harmonic oscillator potential to build the periodic Hamiltonian. The correspondent wave equation is so-called Matheu function. Using the operators of GUP to modify this periodic Hamiltonian, we find the modified Hamiltonian. For obtaining the modified energy levels, we use the perturbation method for Matheu function. Keeping the leading order of the energy levels, we obtain the same energy eigenvalue formation with our previous work [26]. This result shows the minimum observable length equals the compactification radius of the extra dimension.

\section{Acknowledgements}

We are grateful to Houwen $\mathrm{Wu}$ and Zheng Sun for useful discussions. This work is supported in part by NSFC (Grant Nos. 11747171) and Natural Science Foundation of Chengdu University of TCM (Grants Nos. ZRYY1729 and ZRQN1656). Discipline Talent Promotion Program of “Xinglin Scholars” (Grant No. QNXZ2018050) and the key fund project for Education Department of Sichuan (Grant No. 18ZA0173).

\section{Conflicts of Interest}

The authors declare no conflicts of interest regarding the publication of this paper.

\section{References}

[1] Veneziano, G. (1986) A Stringy Nature Needs Just Two Constants. Europhysics Letters, 2, 199. https://doi.org/10.1209/0295-5075/2/3/006

[2] Gross, D.J. and Mende, P.F. (1988) String Theory beyond the Planck Scale. Nuclear Physics B, 303, 407-454. https://doi.org/10.1016/0550-3213(88)90390-2

[3] Amati, D., Ciafaloni, M. and Veneziano, G. (1989) Can Spacetime Be Probed below the String Size? Physics Letters B, 216, 41-47. https://doi.org/10.1016/0370-2693(89)91366-X

[4] Konishi, K., Paffuti, G. and Provero, P. (1990) Minimum Physical Length and the 
Generalized Uncertainty Principle in String Theory. Physics Letters B, 234, 276-284. https://doi.org/10.1016/0370-2693(90)91927-4

[5] Guida, R., Konishi, K. and Provero, P. (1991) On the Short Distance Behavior of String Theories. Modern Physics Letters A, 6, 1487-1503. https://doi.org/10.1142/S0217732391001603

[6] Maggiore, M. (1993) A Generalized Uncertainty Principle in Quantum Gravity. Physics Letters B, 304, 65-69. https://doi.org/10.1016/0370-2693(93)91401-8

[7] Zhao, R., Zhang, L.C., Wu, Y.Q., et al. (2010) Generalized Uncertainty Principle and

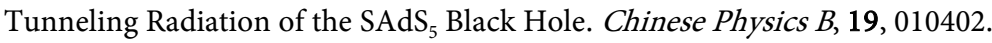
https://doi.org/10.1088/1674-1056/19/1/010402

[8] Corda, C., Chakraborty, S. and Saha, S. (2015) Light from Black Holes and Uncertainty in Quantum Gravity. Electronic Journal of Theoretical Physics, 12, 107.

[9] Haldar, S., Corda, C. and Chakraborty, S. (2018) Tunnelling Mechanism in Noncommutative Space with Generalized Uncertainty Principle and Bohr-Like Black Hole. Advances in High Energy Physics, 2018, Article ID: 9851598. https://doi.org/10.1155/2018/9851598

[10] Mu, B., Wang, P. and Yang, H. (2015) Minimal Length Effects on Tunnelling from Spherically Symmetric Black Holes. Advances in High Energy Physics, 2015, Article ID: 898916. https://doi.org/10.1155/2015/898916

[11] Mu, B.R., Wang, P. and Yang, H.T. (2015) Minimal Length Effects on Schwinger Mechanism. Communications in Theoretical Physics, 63, 715. https://doi.org/10.1088/0253-6102/63/6/715

[12] Gecim, G. and Sucu, Y. (2018) Quantum Gravity Effect on the Hawking Radiation of Charged Rotating BTZ Black Hole. General Relativity and Gravitation, 50, 152. https://doi.org/10.1007/s10714-018-2478-X

[13] Kempf, A., Mangano, G. and Mann, R.B. (1995) Hilbert Space Representation of the Minimal Length Uncertainty Relation. Physical Review D, 52, 1108.

[14] Kempf, A. (1997) Non-Pointlike Particles in Harmonic Oscillators. Journal of Physics $A, 30,2093$. https://doi.org/10.1088/0305-4470/30/6/030

[15] Brau, F. (1999) Minimal Length Uncertainty Relation and Hydrogen Atom. Journal of Physics A, 32, 7691-7696. https://doi.org/10.1088/0305-4470/32/44/308

[16] Brau, F. and Buisseret, F. (2006) Minimal Length Uncertainty Relation and Gravitational Quantum Well. Physical Review D, 74, Article ID: 036002.

https://doi.org/10.1103/PhysRevD.74.036002

[17] Chang, L.N., Minic, D., Okamura, N. and Takeuchi, T. (2002) Exact Solution of the Harmonic Oscillator in Arbitrary Dimensions with Minimal Length Uncertainty Relations. Physical Review D, 65, Article ID: 125027. https://doi.org/10.1103/PhysRevD.65.125027

[18] Dadic, I., Jonke, L. and Meljanac, S. (2003) Physical Review D, 67, Article ID: 087701. https://doi.org/10.1103/PhysRevD.67.087701

[19] Nozari, K. and Azizi, T. (2006) Some Aspects of Gravitational Quantum Mechanics. General Relativity and Gravitation, 38, 735-742. https://doi.org/10.1007/s10714-006-0262-9

[20] Stetsko, M.M. and Tkachuk, V.M. (2006) Perturbation Analysis for Competing Reactions with Initially Separated Components. Physical Review A, 74, Article ID: 012101. https://doi.org/10.1103/PhysRevA.74.012101

[21] Stetsko, M.M. (2006) Corrections to the ns-Levels of Hydrogen Atom in Deformed Space with Minimal Length. Physical Review A, 74, Article ID: 062105. 
[22] Benczik, S.Z. (2007) Investigations on the Minimal-Length Uncertainty Relation. PhD Thesis, Virginia Polytechnic Institute and State University, Blacksburg.

[23] Battisti, M.V. and Montani, G. (2007) The Big-Bang Singularity in the Framework of a Generalized Uncertainty Principle. Physics Letters B, 656, 96-101. https://doi.org/10.1016/j.physletb.2007.09.012

[24] Battisti, M.V. and Montani, G. (2008) Quantum Dynamics of the Taub Universe in a Generalized Uncertainty Principle Framework. Physical Review D, 77, Article ID: 023518. https://doi.org/10.1103/PhysRevD.77.023518

[25] Das, S. and Vagenas, E.C. (2008) Universality of Quantum Gravity Corrections. Physical Review Letters, 101, Article ID: 221301. https://doi.org/10.1103/PhysRevLett.101.221301

[26] $\mathrm{Mu}, \mathrm{B} ., \mathrm{Wu}, \mathrm{H}$. and Yang, H. (2011) The Generalized Uncertainty Principle in the Presence of Extra Dimensions. Chinese Physics Letters, 28, Article ID: 091101.

[27] Antoniadis, I. (1990) A Possible New Dimension at a Few TeV. Physics Letters B, 246, 377-384. https://doi.org/10.1016/0370-2693(90)90617-F

[28] Arkani-Hamed, N., Dimopoulos, S. and Dvali, G.R. (1998) The Hierarchy Problem and New Dimensions at a Millimeter. Physics Letters B, 429, 263-272. https://doi.org/10.1016/S0370-2693(98)00466-3

[29] Antoniadis, I., Arkani-Hamed, N., Dimopoulos, S. and Dvali, G.R. (1998) New Dimensions at a Millimeter to a Fermi and Superstrings at a TeV. Physics Letters $B$, 436, 257-263. https://doi.org/10.1016/S0370-2693(98)00860-0

[30] Randall, L. and Sundrum, R. (1999) A Large Mass Hierarchy from a Small Extra Dimension. Physical Review Letters, 83, 3370-3373. https://doi.org/10.1103/PhysRevLett.83.3370

[31] Randall, L. and Sundrum, R. (1999) An Alternative to Compactification. Physical Review Letters, 83, 4690-4693. https://doi.org/10.1103/PhysRevLett.83.4690

[32] Dvali, G.R., Gabadadze, G. and Porrati, M. (2000) 4D Gravity on a Brane in 5D Minkowski Space. Physics Letters B, 485, 208-214. https://doi.org/10.1016/S0370-2693(00)00669-9

[33] Appelquist, T., Cheng, H.C. and Dobrescu, B.A. (2001) Bounds on Universal Extra Dimensions. Physical Review D, 64, Article ID: 035002. https://doi.org/10.1103/PhysRevD.64.035002

[34] Cremades, D., Ibanez, L.E. and Marchesano, F. (2002) Standard Model at Intersecting D5-Branes: Lowering the String Scale. Nuclear Physics B, 643, 93-130. https://doi.org/10.1016/S0550-3213(02)00746-0

[35] Kokorelis, C. (2004) Exact Standard Model Structures from Intersecting D5-Branes. Nuclear Physics B, 677, 115-163. https://doi.org/10.1016/j.nuclphysb.2003.11.007

[36] Li, Z.G. and Ni, W.T. (2008) Extra Dimensions and Atomic Transition Frequencies. Chinese Physics B, 17, 70-75.

[37] Bezerra, V.B. and Rego-Monteiro, M.A. (2004) Some Boundary Effects in Quantum Field Theory. Physical Review D, 70, Article ID: 065018.

[38] Takenaga, K. (2000) Quantization Ambiguity and Supersymmetric Ground State Wave Functions. Physical Review D, 62, Article ID: 065001. https://doi.org/10.1103/PhysRevD.62.065001

[39] Doncheski, M.A. and Robinett, R.W. (2003) Wave Packet Revivals and the Energy Eigenvalue Spectrum of the Quantum Pendulum. Annals of Physics, 308, 578-598. https://doi.org/10.1016/S0003-4916(03)00171-4

[40] McLachlan, N.W. (1947) Theory and Application of Mathieu Functions. Clarendon 
Press, Oxford.

[41] Hradil, et al. (2006) Minimum Uncertainty Measurements of Angle and Angular Momentum. Physical Review Letters, 97, Article ID: 243601.

[42] Abramowitz, M. and Stegun, I.A. (1972) Handbook of Mathematical Functions: With Formulas, Graphs, and Mathematical Tables. Dover Publications, New York, 724. 